\title{
Rettungsarzt bei der Air Zermatt
}

\section{Tanja Kühnle}

Dipl.-Biol., Managing Editor

\author{
Die letzte Prüfung ist geschafft, das Medizinstudium beendet. Und wie geht es \\ jetzt weiter? In unserer neuen Serie «Du findest Deinen Weg!» stellen wir Ihnen in \\ unregelmässigen Abständen aussergewöhnliche Berufsperspektiven für Mediziner \\ vor. In der ersten Ausgabe berichtet Dr. Axel Mann von seiner Tätigkeit als Leiten- \\ der Rettungsarzt bei der Air Zermatt.
}

\section{Herr Mann, wie kam es, dass Sie Rettungsarzt bei der Air Zermatt geworden sind?}

Ich war bereits während meiner Facharztausbildung von 1978 bis 1983 in Deutschland in der Luftrettung tätig. Meine Zuneigung und Liebe zum Bergsport, die Pionierleistungen der Air Zermatt, die weit über die Grenzen der Schweiz bekannt waren, und die Kombination aus Technik und Fliegerei im Hochgebirge veranlassten mich 1983 zu einer Vorstellung bei der Air Zermatt. Mein damaliger Chef im Spital stellte mich für die Tätigkeit befristet frei. 1985 wurde mir dann die Ärztliche Leitung bei der Air Zermatt übertragen.

\section{Wie viele Ärzte sind für die Air Zermatt tätig?}

Die Air Zermatt hatte bereits 1973 einen festen Arzt auf der Basis. Mittlerweile haben wir einen Pool von 41 Fachärzten Anästhesie, die den Dienst übers Jahr für 2-4 Wochen auf den Basen in Zermatt und Raron verrichten. Normalerweise arbeiten unsere Ärzte als Anästhesisten oder Intensivmediziner in Zentrumsspitälern. Das hat den Vorteil, dass sie auf diesem Gebiet stets in Übung und auf dem neuesten Stand bleiben, Notarzt- und Heli-

\section{Voraussetzungen für die Tätigkeit als Rettungsarzt}

\section{bei der Air Zermatt AG}

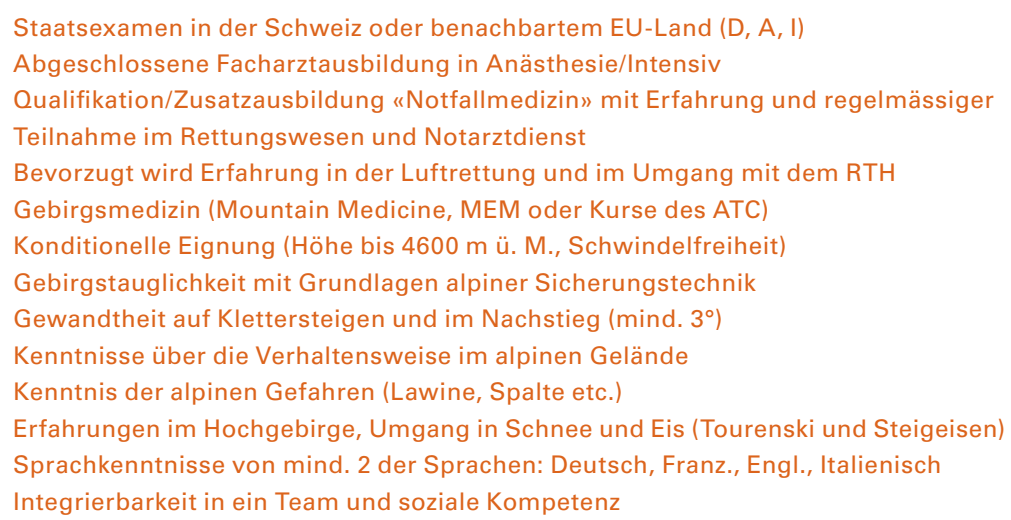

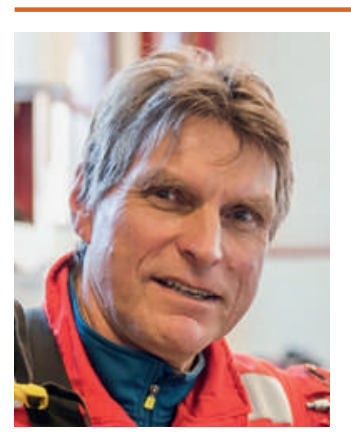

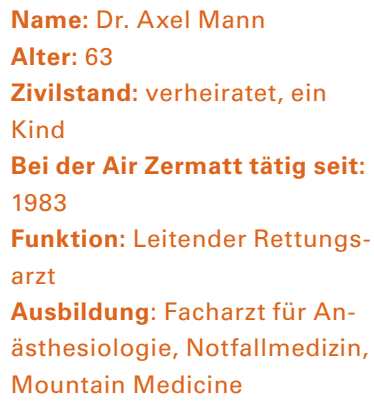

koptererfahrung beibehalten und mit Motivation und Engagement nach Zermatt kommen, um die anspruchsvolle Gebirgsrettung im Kontrast zum Klinikalltag zu erleben.

\section{Was fasziniert Sie an Ihrem Beruf am meisten?}

Die Faszination besteht aus einer Mischung aus Hochgebirge, Fliegerei und Notfallmedizin - der Verbindung zwischen Beruf und Hobby. Jeder Einsatz ist individuell und verlangt bei unserem Einsatzspektrum ein hohes technisches Verständnis und eine gezielte Bergungstechnik, manchmal auch Improvisation. Es erfüllt mich abends nach erfolgreichen Einsätzen mit Genugtuung, einen Notfallpatienten oder Verunglückten im Team adäquat versorgt und ins geeignete Spital zur weiteren Versorgung transportiert zu haben.

\section{Das Telefon klingelt, der nächste Einsatz ruft. Wie} bereiten Sie sich auf das vor, was Sie vielleicht erwartet? Es ist nur selten möglich, sich auf einen Patienten einzustellen. Bei Skiunfällen und noch gravierender im Sommer bei Gebirgsunfällen wird das Verletzungsoder Erkrankungsmuster in der Alarmierung oft sehr ungenau gemeldet. Nähere Informationen erhalten wir über Funk im Winter durch den Pistenpatrouilleur. Die genaue Lokalisation des Einsatzortes ist wichtig. Der Pilot hat generell drei Möglichkeiten, den Notarzt und 


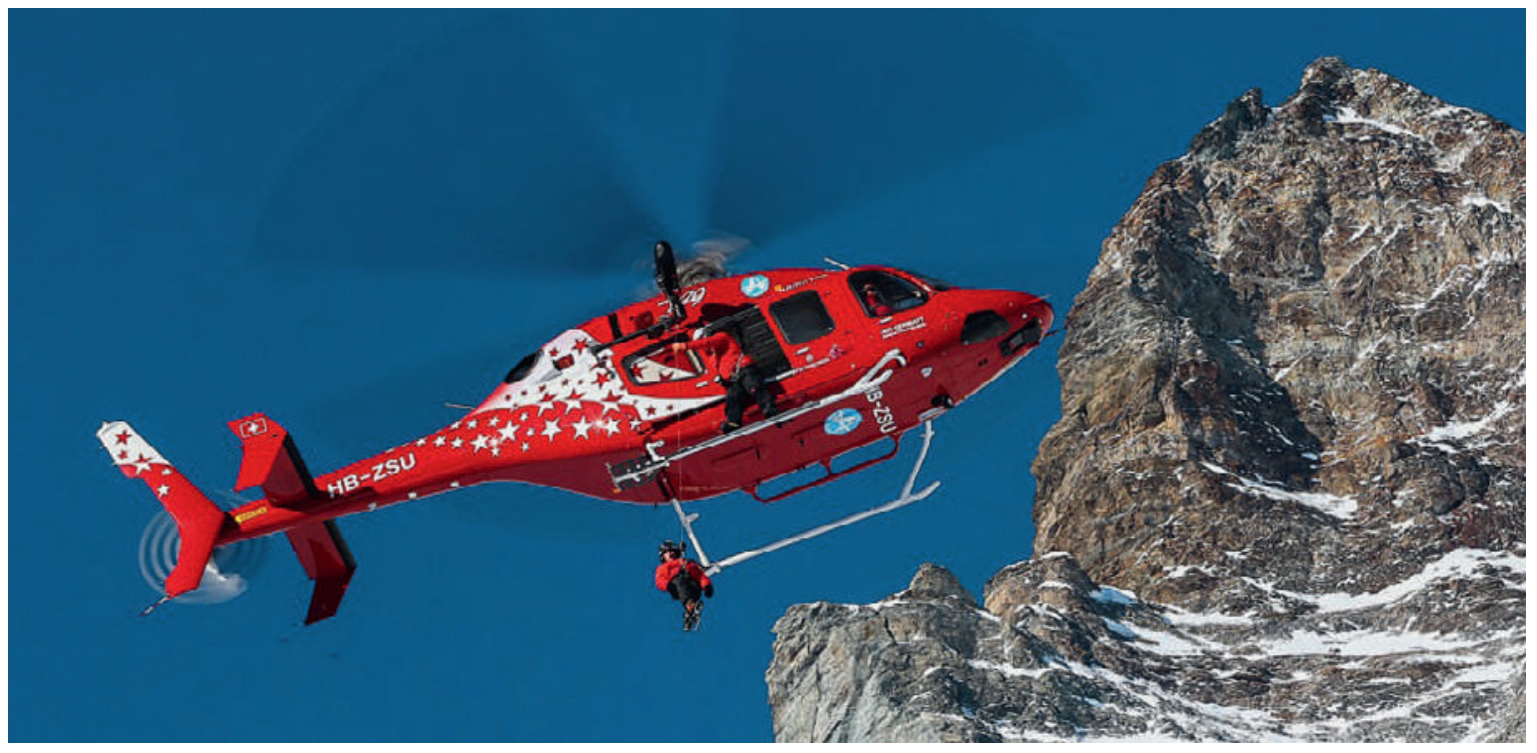

Windenaktion am Matterhorn.

eventuell Rettungssanitäter zum Patienten zu bringen: Landung an der Unfallstelle, schwebendes Aussteigen oder eine Windenoperation. Bei der Windenoperation ist der Rettungssanitäter gleichzeitig Windenoperator und lässt den Notarzt mit dem erforderlichen Equipment (Bergesack mit Vakuummatratze und Notarztrucksack) mit der Rettungswinde maximal 90 Meter zum Patienten ab. Für grössere Höhen gibt es noch das Long-Line-Verfahren, mit dem der Retter bis zu 200 Meter am fixen Seil unter dem Helikopter hängend an die Unfallstelle geflogen werden kann.

Wie stelle ich mir einen typischen Rettungseinsatz vor? Den «typischen" Rettungseinsatz gibt es selbst im Winter nicht. Die Lokalisation und die Landemöglichkeit variieren, ebenso die Meteosituation mit Windverhältnissen sowie die Höhe und damit auch die Leistungsfähigkeit des Helikopters. Selbst bei gleichem Verletzungsmuster ist durch die Umgebungsbedingungen und bergungstechnische Anforderung kaum eine Standardsituation realisierbar.

\section{Zu wie vielen Einsätzen werden Sie am Tag gerufen?}

Die Anzahl der Rettungseinsätze pro Tag ist saison- und wetterabhängig. Hochsaison ist in der Weihnachtszeit,

\section{Über die Air Zermatt AG}

Die Air Zermatt AG wurde 1968 gegründet. Auf den drei Basen in Gampel, Raron sowie Zermatt sind rund 65 festangestellte Mitarbeiter tätig. Die insgesamt neun Helikopter mit den rot-weissen Sternen sind weltweit bekannt. Die Air Zermatt rettet Verunfallte von Bergen oder aus Gletscherspalten und leistet Transport- sowie Touristenflüge. Ihren grossen Erfahrungsschatz geben die Bergretter am Air Zermatt Training Center (ATC) an Profis und Laien weiter. Gemeinsam mit der Rettungsstation Zermatt hat die Air Zermatt im April 2011 zudem im Himalaya eine Rettungsstation aufgebaut. an Fasnacht und Ostern. An einem Skiwochenende mit guten Bedingungen fielen kürzlich 41 Einsätze an, welche wir mit vier Rettungshubschraubern (RTHs) bewältigten. Im gesamten Jahr fliegt die Air Zermatt ca. 1800 Rettungseinsätze, wobei zwei Basen ganzjährig rund um die Uhr mit zwei RTHs besetzt sind. In der Wintersaison deckt ein dritter RTH die Spitzenbelastungen ab.

\section{Sind Sie auch nachts im Einsatz?}

Nachts haben wir keine Präsenz auf der Basis, sondern Rufdienst von Zuhause, sind aber in der Regel 20 Minuten nach dem Alarm in der Luft. Die Entscheidung, einen Einsatz nachts durchzuführen, treffen der Notarzt medizinisch und der Pilot fliegerischerseits. Selbst bei guten Sichtverhältnissen, z.B. bei Vollmond, ist das Risiko nachts deutlich höher als tagsüber. Die Piloten absolvieren ein spezielles Training und arbeiten mit Nachtsichtgeräten. Oftmals verbindet die 144 den Anrufer oder Spitalarzt direkt mit unserem Notarzt, um die Indikation abzuklären.

\section{Fliegt die Air Zermatt ausschliesslich im Wallis?}

Wir fliegen auch im angrenzenden Italien das Spital in Aosta an und versorgen die grenznahen Regionen, wenn der italienische RTH unterwegs ist. Wir haben eine enge Kooperation mit der Air Glacier und Rega. Seltene Einsätze führen uns in andere Kantone, wie ins Tessin.

\section{Die Air Zermatt unterstützt die Bergretter in Nepal. Waren Sie auch schon einmal vor Ort?}

Ich kenne die Himalaya-Region aus dem Jahr 2000, da existierte die Kooperation noch nicht. Es ist mit Sicherheit eine grosse Aufgabe, die Infrastrukturen für die Rettungstechnik und die Notfallmedizin aufzubauen. 


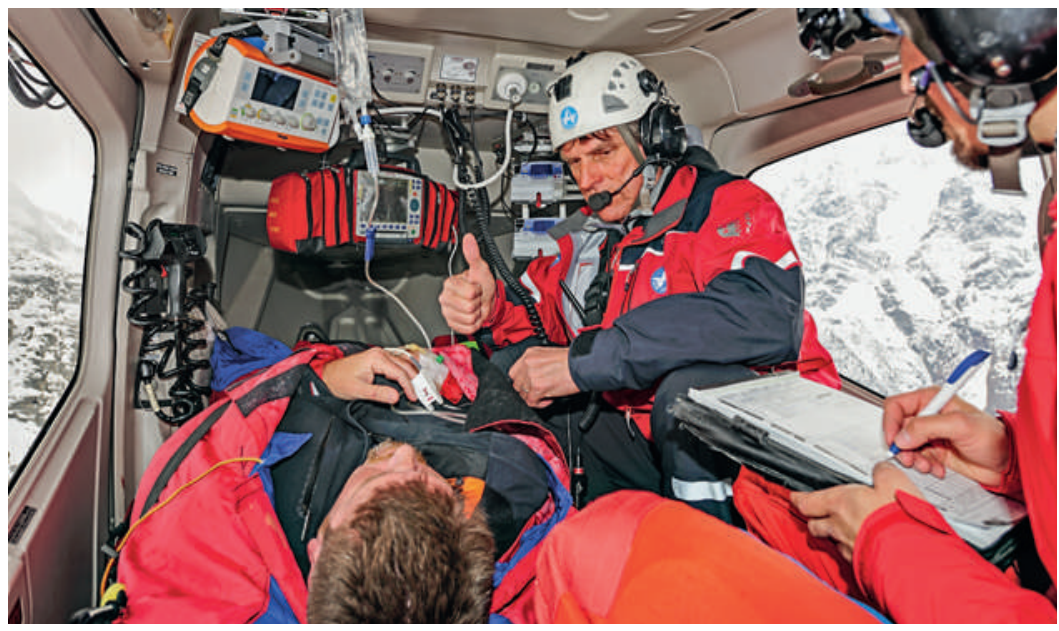

Axel Mann signalisiert seinem Patienten, dass alles in Ordnung ist.

Waren Sie schon einmal im Ausland im Einsatz? Seit 1978 bin ich in der Rettungsfliegerei in Deutschland engagiert und war sechs Jahre für die Formel 1 und 15 Jahre für nationale und internationale Motorradrennläufe als Rennarzt unterwegs und verantwortlich.

\section{Was ist das häufigste Krankheitsbild, zu dem Sie gerufen werden?}

Häufige internistische Krankheitsbilder sind «Akutes Kardiales Syndrom», "Zerebrovaskuläre Insuffizienz» und Verlegungen der Regionalspitäler in Zentren der Maximalversorgung.

Haben Sie manchmal Angst, dass Ihnen bei einem Einsatz selbst etwas passiert?

Angst wäre im Einsatz ein schlechtes Gefühl, sie blockiert eine situationsgerechte Analyse der Gefahrensituation und eine konsequente Verhaltensweise zu deren Abwehr. Jedoch sollte man den Respekt vor dem alpinen Gelände, dessen Gefahren und auch vor der Fliegerei im Grenzbereich nie verlieren. In einer speziellen Situation kann durchaus ein Angstgefühl auftreten; sollte ein Notarzt aber bereits vor dem Einsatz Angst verspüren, hat er das verkehrte Beschäftigungsfeld gewählt.

\section{Was ist die grösste Herausforderung bei einem Einsatz?}

Bei Hochgebirgseinsätzen im Sommer wie auch bei Gletscherspalteneinsätzen kann die persönliche konditionelle Leistungsgrenze erreicht werden. Das Verletzungsmuster kann durchaus im Sinne eines schweren Polytraumas ausgeprägt sein, so dass eine Primärversorgung vor Ort erforderlich wird; teils nur mit Assistenz eines Bergführers oder Rettungsspezialisten, welcher technisch, aber weniger medizinisch versiert ist. ein Abtransport z.B. aus der Felswand evaluiert werden. In solchen Extremsituationen kann auch mal Improvisation erforderlich sein.

\section{Können Sie den Helikopter im Notfall auch selbst fliegen?}

Es gibt einen Spruch: «Schuster, bleib bei deinen Leisten.» Unserer Piloten haben eine langjährige Ausbildung sowie diverse Trainings absolviert und arbeiten hochprofessionell. Ihre Fähigkeiten sind unsere Lebensversicherung, denn das Fliegen und Landen im Hochgebirge ist häufig eine Herausforderung. Trotzdem bereitet es mir Spass und Freude, bei eingebautem Doppelsteuer, nach einem Einsatz vom Spital zurück zur Basis fliegen zu dürfen. Man benötigt schon ein feinmotorisches Geschick und Übung, um auf der Plattform des Dachlandeplatzes starten und landen zu können. Da unsere Piloten die Fluglehrerberechtigung besitzen, ist die Aktion auch legitimiert.

\section{Lässt sich Ihr Beruf gut mit der Familie vereinbaren?}

Um die erforderliche Flexibilität gewährleisten zu können, muss die Partnerin sehr tolerant und verständnisvoll sein für teils unvermutet auftretende berufliche Anforderungen. Auch nach schwierigen Einsätzen oder belastungsintensiven Tagen sollte sie den ruhenden Pol darstellen und private Probleme möglichst fernhalten. Ohne grosse Unterstützung seitens der Familie ist eine so hohe Leistung und Konzentration nicht zu erbringen.

\section{Ihr Ratschlag für junge Kollegen, die auch in der Luftrettung tätig werden wollen:}

Die Luftrettung mit dem Arbeitsgerät «Helikopter» stellt ein gewisses Gefahrenpotential per se dar. Wir sind in der dritten Dimension. Erschwert wird dies noch durch die Unzugänglichkeiten des Hochgebirges, die Bergungstechnik per Winde oder Long Line, die Improvisationsnotwendigkeit sowie durch die Anforderung an die eigene Kondition. Ein Verkehrsanfänger würde seine Fahrprüfung auch nicht auf einem Formel-Rennwagen absolvieren; in Analogie verlangen wir von unseren Notärzten Helikoptererfahrung auf Flachland- oder Mittelgebirgsbasen, um den Umgang mit dem Helikopter nicht direkt unter Extrembedingungen erfahren zu müssen. Ausführliche Informationen zu den Voraussetzungen gibt es auf unserer Homepage unter www.air-zermatt. ch/wordpress/bewerbung-als-rettungsarzt/

Bildnachweis

Air Zermatt AG

Haben Sie als Mediziner auch einen aussergewöhnlichen Beruf, den Sie unseren Lesern gern vorstellen möchten? Dann freuen wir uns auf Ihre Bewerbung per E-Mail an: tkuehnle[at]emh.ch 